\title{
Anisotropic modeling of structural components using embedded crystal plasticity constructive laws within finite elements
}

\author{
Marko Knezevic ${ }^{\mathrm{a}, *}$, Justin Crapps ${ }^{\mathrm{b}}$, Irene J. Beyerlein ${ }^{\mathrm{c}}$, \\ Daniel R. Coughlin ${ }^{\mathrm{d}}$, Kester D. Clarke ${ }^{\mathrm{d}}$, Rodney J. McCabe ${ }^{\mathrm{d}}$ \\ ${ }^{a}$ Department of Mechanical Engineering, University of New Hampshire, Durham, NH 03824, USA \\ ${ }^{\mathrm{b}}$ Mechanics of Materials Section, ExxonMobil Upstream Research Company, Houston, TX, 77098, USA \\ ${ }^{c}$ Theoretical Division, Los Alamos National Laboratory, Los Alamos, NM 87545, USA \\ ${ }^{\mathrm{d}}$ Materials Science and Technology Division, Los Alamos National Laboratory, Los Alamos, NM 87545, USA
}

\begin{abstract}
Pins are commonly used to join members of mechanical mechanisms. In order to maintain the integrity of the joint and prevent failure, there must be sufficient material of adequate strength around the pin hole to sustain the bearing and tear out loads from the pin connection. In this work, a multi-scale materials simulation model based on finite elements (FE) is developed for design and evaluation of materials for this application. We specifically examine several constitutive models for simulating the elasto-plastic behavior of the plate material while maintaining computational efficiency. Here, models are developed for two plate materials: copper $(\mathrm{Cu})$ and $\alpha$-uranium ( $\alpha-\mathrm{U})$, with vastly different plastic behaviors owing to their crystal structures and crystallographic textures. For $\mathrm{Cu}$, digital image correlation (DIC) tests are carried out during loading of the plate/pin assembly to characterize the strain distributions in the critical hole/pin area. The corresponding FE simulations are carried out using a combination of constitutive laws involving a fine-scale polycrystal plasticity calculation, a J2 flow theory, or a combination of both. We show that the FE model using the fine-scale polycrystal plasticity constitutive law successfully captures the DIC strain fields in the hole region at different plate displacements. Surprisingly, use of the more computationally efficient J2 plasticity model also produces reasonable results in comparison with the measurements and the fine-scale constitutive law. An interesting finding is that combining fine-scale constitutive laws in the region surrounding the hole and continuum $\mathrm{J} 2$ theory elsewhere gives the worst agreement. It also precariously produces non-conservative estimates for the hole opening with applied displacement. These results on $\mathrm{Cu}$ helped subsequent simulations on $\alpha-\mathrm{U}$, where use of the finescale polycrystal simulation is fundamental considering the highly plastic anisotropic response of this complicated material. We demonstrate that in the $\alpha-\mathrm{U}$ plates, the localized deformation in the hole region is highly dependent on the direction of displacement.
\end{abstract}

Keywords: Copper, Uranium, Constitutive Modeling, Finite Element Method, Texture

\footnotetext{
* Corresponding author at: University of New Hampshire, Department of Mechanical Engineering, 33 Academic Way, Kingsbury Hall, W119, Durham, New Hampshire 03824, United States. Tel.: 603862 5179; fax: 603862 1865.

E-mail address: marko.knezevic@unh.edu (M. Knezevic).
} 


\section{Introduction}

Rivets, bolts, and pins are frequently used to join engineering components for which the joint is loaded with shearing and bearing stresses [1,2]. The geometries of these types of joints can be complicated such as in the rivet bonded sheets of an aircraft skin or the joints between members of a bridge. These bonds can be idealized as two or more sheets of metal joined by at least one cylindrical pin (pin will be used from here on to describe the cylindrical portion of the rivet, bolt, or pin joining two or more plates). Traditionally, these joints are analyzed by assuming a point force acting at the joint to calculate applied stresses that are then used to design joint geometries with sufficient engineering safety factors. The usual loading state places the pins in a state of shear stress and the components held together in a state of normal bearing stress and tear out shear stress. The components under consideration of the present work are idealized as two outer plates and one inner plate in order to minimize potential for bending in the joint (Fig. 1). When the plates are pulled apart, the deformation is concentrated in the plate/pin intersection, and failure is likely to occur in the material surrounding the hole. For polycrystalline metal plates, the local stress-strain response is sensitive to both the heterogeneous nature of the loading and the microstructure. The material in the region around the hole experiences very different deformation than in the plate away from the hole. At the same time, some metals and their alloys used in engineering applications such as magnesium [3-5], titanium [6, 7], zirconium [8,9], beryllium [10,11], or uranium [12-14] considered here exhibit significant stress/strain anisotropy and tension-compression asymmetry related to microstructure. Therefore, coupling between the development of non-uniform deformation and the local deformation response of the material are needed for optimal designs of joints.

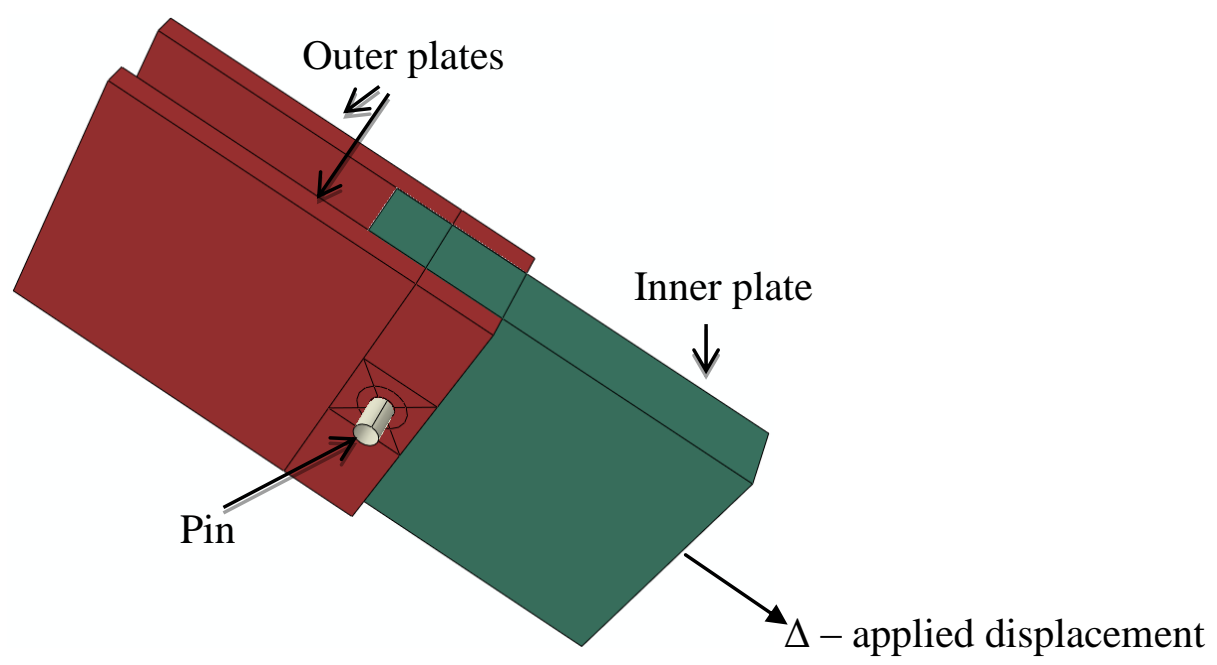

Figure 1 Structural component assembly consisting of two outer plates of length $76.2 \mathrm{~mm}$, thickness $3.2 \mathrm{~mm}$, and width $38 \mathrm{~mm}$; an inner plate of length $76.2 \mathrm{~mm}$, thickness $8.9 \mathrm{~mm}$, and width $38 \mathrm{~mm}$; and a $3.2 \mathrm{~mm}$ diameter pin. The overlap of the plates is $18 \mathrm{~mm}$. Edge of the square insert is $10 \mathrm{~mm}$. The inner plate is pulled in the axial direction while the outer plates are fixed.

We study the material deformation response, particularly near the hole region, while the joined plates are being pulled apart in a direction perpendicular to the pin axis. We build a multiscale 
finite element (FE) model to study the development of stress and strain in the plate assembly, particularly in the pin/plate intersection. Two different materials are considered, polycrystaline copper $(\mathrm{Cu})$ and $\alpha$-uranium $(\alpha-\mathrm{U})$, as they represent a material with relatively simple deformation behavior and a material with complex deformation behavior, respectively. The two metals have different crystal structures and exhibit different plastic behavior. $\mathrm{Cu}$ has a face center cubic (FCC) crystal structure and, to plastically deform, it utilizes one slip mode that contains 12 crystallographically identical slip systems. It can accommodate deformation applied in any direction using a combination of slip systems within the same mode, and hence is viewed approximately as plastically 'isotropic'. $\alpha-U$ has an orthorhombic crystal structure and utilizes four unique slip systems and up to four unique twinning modes that are not crystallographically identical [15-17]. These modes require different activation stresses and their activation stresses are very sensitive to crystal orientation $[13,18]$. Deformation applied in different directions will not use the same combination of slip modes. Consequently, response of $\alpha-\mathrm{U}$ is highly elastically and plastically anisotropic.

While it has been common practice to choose among a variety of deformation models for $\mathrm{Cu}$ [1921], the same is not true for $\alpha-U$ [13]. Such complicated plastic behavior is not well represented by continuum plasticity theories and requires a constitutive law based on polycrystal plasticity able to represent various microstructural complexities influencing the overall material behavior $[22,23]$. Polycrystal plasticity models have been used within FE codes to simulate compression/tension tests [24, 25], bending [26, 27], torsion [28], springback [29, 30], cupdrawing [31-34], sheet hydroforming [35], bulk forming [36-39], biaxial deformation [40] and axial crush response [41] or used to calibrate continuum level models for example for stamping simulation [42]. These studies have demonstrated the remarkable success of polycrystal models in predicting the anisotropic mechanical response as well as the evolution of the underlying microstructure during large plastic deformation [43-45]. A major drawback of these modes is their computational cost that prevents their broader adoption as a simulation tool at macroscopic levels. Several approaches have been explored to accelerate polycrystal plasticity models within FE including the adaptive sampling [46], the spectral solvers [47-54], a specialized hardware [55-57] and microstructure data compaction [58,59]. To consider various types of plate material, such as both $\mathrm{Cu}$ and $\alpha-\mathrm{U}$, while using the same multi-scale framework, here we enable multiple constitutive laws to maintain computational efficiency while achieving mechanical accuracy [60]. In this work, we adopt isotropic elasticity, isotropic J2 plasticity [61], and a polycrystal plasticity based on the self-consistent homogenization scheme [62,63].

For comparison to the model, we involve experimental testing of the plate assembly for $\mathrm{Cu}$. A mock plate/pin assembly was built consisting of two $\mathrm{Cu}$ plates joined by a single pin of hardened steel. The assembly is displaced along the plate direction, as indicated in Fig. 1. We use digital image correlation (DIC) to characterize the 2D strain fields in-situ during loading. This is accomplished by applying a black and white speckle pattern to the area of interest (hole region) on the mock plate/pin using standard spray paint. We perform DIC by using Correlated Solutions software [64]. We find that the FE model that uses the fine-scale polycrystal plasticity constitutive law successfully captures the DIC strain fields in the hole region at different plate displacements. For this case, we also show that computationally efficiency can be greatly improved without sacrificing accuracy by reducing the number of grains used in the polycrystal calculation. The FE-VPSC simulations were carried out for $\alpha-U$ plates. It was shown that in $\alpha-U$ 
plate, the localized deformation in the hole region is highly dependent on the direction of displacement relative to the orientation of the plate.

\section{Multi-scale model}

To calculate the strain and stress distributions around the hole and overall mechanical response of the plate assembly, we employ a multi-scale (FE) model extending from the microscale to the macroscale. At the macro-scale, we model the deformation of the plate assembly using finite elements. At the meso-scale, we use multiple types of constitutive laws, ranging from simple isotropic elasticity to a complex, polycrystal model. At the microscale, for activating plastic slip within each crystal within the polycrystal model, we used an extended Voce type [65] hardening model for $\mathrm{Cu}$ [66] and a dislocation density-hardening model for $\alpha-\mathrm{U}$ [13, 67, 68]. For twinning, we use a different model that incorporates both the lattice reorientation and shear provided by the twin. These latter refinements regarding dislocation densities and twinning are only operative when the polycrystal VPSC model is used as the constitutive law. In the multi-scale model, all components mentioned above, the macro-meso-micro models, act concurrently. These have also been detailed in prior works $[37,69,70]$. For the constitutive response at each integration point, we used one or a combination among three models: isotropic elasticity, isotropic J2 plasticity, and a polycrystal plasticity model called the viscoplastic self-consistent (VPSC) scheme. These three models are discussed in turn below.

\subsection{Isotropic models}

This method is the conventional FE based method, which uses a Young's modulus and a Poisson's ratio for the elastic response and the flow stress data for the J2 plasticity response of the material at each integration point [61]. Young's modulus and a Poisson ratio for $\alpha-\mathrm{U}$ are 208 $\mathrm{GPa}$ and 0.23 and for $\mathrm{Cu}$ are $117 \mathrm{GPa}$ and 0.33 , respectively. These materials have been characterized using experimental uniaxial compression and tension. Figures 2 and 3 show the data in compression. These curves were used in $\mathrm{J} 2$ isotropic plasticity simulations as well as for the calibration of the hardening parameters for FE-VPSC.

\subsection{VPSC polycrystal model}

The VPSC model provides the constitutive response of each point based on rate-sensitive plasticity. The elastic response is neglected. In VPSC, a polycrystal is modeled by a collection of grains, each with a given crystallographic orientation, shape and volume fraction. As with all polycrystalline plasticity models, VPSC calculations relate the deformation of the constituent grains to that of the polycrystal. To calculate the interaction of a grain with the other grains in the polycrystal, it is treated as a visco-plastic inclusion embedded in a homogeneous effective medium (HEM). A Green's function approach is used to solve the inclusion problem [62]. The anisotropic plastic properties of the surrounding HEM is equal to the averaged anisotropic plastic properties of all grains, and hence, the term "self-consistent" in the technique. The HEM response is the deformation response of the material point in the FE simulation.

VPSC uses crystal plasticity theory to relate the deformation of a representative grain to its active slip and twinning modes. Each grain is allowed to deform by a combination of slip and deformation twinning modes, where the active systems and the distribution of shear are 
predicted. The slip or twin shearing rate, $\dot{\gamma}^{k}$, on a system, $k$, is related to the resolved shear stress, $\tau^{S}$, on that system via a visco-plastic flow rule given by:

$$
\dot{\gamma}^{k}=\dot{\gamma}_{0}\left|\frac{\tau^{k}}{\tau_{c}^{k}}\right|^{\frac{1}{m}} \operatorname{sign}\left(\tau^{k}\right)
$$

where $\dot{\gamma}_{0}$ is the reference slip rate, $m$ is the rate sensitivity parameter, and $\tau_{c}^{k}$ is the threshold stress on the system. In the present calculations, $\dot{\gamma}_{0}$ is set to be the macroscopic strain rate and $1 / m$ equals 10 . The slip and twinning modes made available to the grains are provided a priori depending on the material. The calculation, however, determines which ones are active.

The flow law Eq. (1) introduces threshold stresses, $\tau_{c}^{k}$, for activating the available slip and twinning systems. These threshold stresses, $\tau_{c}^{k}$, generally evolve or "harden" as a function of strain. While the same hardening model may be used for all slip systems, the actual value of $\tau_{c}^{S}$ may differ for each slip system and from grain to grain in the polycrystal due to a difference in slip activity and strain levels. Because their plastic behaviors exhibit different levels of complexity, we will employ different hardening models for $\mathrm{Cu}$ and $\alpha-\mathrm{U}$. These will be detailed further in the next section.

At the polycrystal level, the use of a rigid-viscoplastic approach implies that the constitutive relations at single-crystal level can be expressed in terms of the Cauchy stress deviator, $\boldsymbol{\sigma}^{\prime}$, and the viscoplastic strain-rate, $\dot{\boldsymbol{\varepsilon}}_{\mathrm{vp}}[1]$ :

$$
\dot{\boldsymbol{\varepsilon}}_{v p}(\mathbf{x})=\sum_{k=1}^{N_{k}} \mathbf{m}^{k}(\mathbf{x}) \dot{\gamma}^{k}(\mathbf{x})
$$

where the sum runs over all $N_{k}$ slip and twin systems and $\mathbf{m}^{k}$ is the Schmid tensor associated with slip or twinning system $k$. A linear relation (an approximation of the actual local nonlinear relation, Eq. 2) is assumed between $\dot{\boldsymbol{\varepsilon}}_{\mathrm{vp}}^{(\mathrm{r})}$ and $\boldsymbol{\sigma}^{\prime(\mathrm{r})}$, i.e.

$$
\dot{\boldsymbol{\varepsilon}}_{\mathrm{vp}}^{(\mathrm{r})}=\boldsymbol{M}^{(\mathrm{r})}: \boldsymbol{\sigma}^{(\mathrm{r})}+\dot{\boldsymbol{\varepsilon}}^{\mathrm{o}(\mathrm{r})}
$$

where $\boldsymbol{M}^{(\mathrm{r})}$ and $\dot{\boldsymbol{\varepsilon}}^{\mathrm{O}(\mathrm{r})}$ are the linearized viscoplastic compliance and back-extrapolated strainrate of grain (r), respectively. The behavior of the single crystals can be homogenized assuming a linear relation at the effective medium (polycrystal, $p x$ ) level:

$$
\dot{\boldsymbol{\varepsilon}}_{\mathrm{vp}}^{(\mathrm{px})}=\boldsymbol{M}^{(\mathrm{px})}: \boldsymbol{\sigma}^{(\mathrm{px})}+\dot{\boldsymbol{\varepsilon}}^{\mathrm{o}(\mathrm{px})}
$$

where $\dot{\boldsymbol{\varepsilon}}_{\mathrm{vp}}^{(\mathrm{px})}$ and $\boldsymbol{\sigma}^{(\mathrm{px})}$ are the effective (polycrystal) deviatoric strain-rate and stress tensors and $\boldsymbol{M}^{(\mathrm{px})}$ and $\dot{\boldsymbol{\varepsilon}}^{\mathrm{o}(\mathrm{px})}$ are the tangent viscoplastic compliance and back-extrapolated strain-rate of an a priori unknown homogeneous medium that represents the behavior of the polycrystal. The usual procedure to obtain the homogenized response of a linear polycrystal is the linear selfconsistent method. The VPSC code contains several options for linearization schemes starting from the upper bound secant to the intermediate affine and $n_{\text {eff }}$ and finally to the lower bound tangent scheme. While any choice of linearization would operate within the FE-VPSC code, the simulation results presented in this paper were based on the $n_{\text {eff }}=10$ scheme. We selected this scheme for consistency with the earlier simulations involving $\alpha$-U [13] from where the hardening parameters were taken. The problem underlying the self-consistent method is that of a single 
crystal $r$ of moduli $\boldsymbol{M}^{(\mathrm{r})}$ and $\dot{\boldsymbol{\varepsilon}}^{\mathrm{o}(\mathrm{r})}$, embedded in an infinite medium of moduli $\boldsymbol{M}^{(\mathrm{px})}$ and $\dot{\boldsymbol{\varepsilon}}^{\mathrm{o}(\mathrm{px})}$, which can be obtained using a standard self-consistent analysis.

The above numerical scheme can be used to predict the stress-strain response and the microstructure evolution of the polycrystal (crystallographic and morphological texture and hardening evolution) by applying the viscoplastic deformation to the polycrystal in incremental steps. The latter is performed assuming constant rates during a time interval $\Delta t$ and using the strain-rates $\dot{\boldsymbol{\varepsilon}}_{\mathrm{vp}}^{(\mathrm{r})}$ and rotation-rates $\dot{\boldsymbol{\omega}}^{(\mathrm{r})}$ (times $\Delta \mathrm{t}$ ) to update the shape and orientation of the grains. The shear rates (times $\Delta t$ ) are used to update the critical stress of the deformation systems due to strain hardening after each deformation increment.

\subsection{Materials and associated hardening models}

The $\mathrm{Cu}$ material is a sheet material of oxygen free high-conductivity $\mathrm{OFHC} \mathrm{Cu}$. It had been conventionally rolled and annealed. The $\alpha-U$ material used in this study is the same straight rolled $\alpha-U$ as reported in [13]. It was vacuum induction cast in ingots, rolled in multiple steps with intermediate anneals before reaching a final thickness of $8 \mathrm{~mm}$. The grains were equi-axed with an average grain size of $15 \mu \mathrm{m}$ and free of twins. Uniaxial compression stress-strain test were performed on $\mathrm{Cu}$ and used to characterize the model parameters associated with the VPSC constitutive law. The data and constitutive parameters for $\alpha$-U were taken from the prior work [13].

\subsubsection{Model for $\mathrm{Cu}$}

Figure 2 shows the measured stress-strain curves for compression in the three orthogonal directions of the sheet, the normal direction (ND), the transverse direction (TD), and the rolling direction (RD). We use the VPSC for $\mathrm{Cu}$ to simulate these curves using the same set of hardening parameters. The slip systems made available were the $12\{111\}\langle 110\rangle$, the common slip mode for $\mathrm{Cu}$ at room temperature. Hardening of the threshold stresses, $\tau_{c}^{k}$, for activating these slip systems, $k$, was assumed to follow a Voce law, which is given by [66]:

$$
\tau_{c}^{k}=\tau_{o o}^{k}+\left(\tau_{1}^{k}+\theta_{1}^{k} \Gamma\right)\left(1-\exp \left(-\Gamma^{(r)}\left|\frac{\theta_{o}^{k}}{\tau_{1}^{k}}\right|\right)\right)
$$

where $\Gamma$ is the accumulated shear per grain and $\tau_{o o}^{k}, \tau_{1}^{k}, \theta_{o}^{k}$ and $\theta_{1}^{k}$ are the initial slip resistance, initial hardening rate, asymptotic hardening rate and back-extrapolated slip resistance, respectively.

In the VPSC simulation, the boundary conditions were strain imposed in the direction of loading and stress-free boundary conditions on the lateral faces. The imposed strain rate was $0.001 / \mathrm{s}$, as in the mechanical tests.

Figure 2 also compares the calculated stress-strain curves in compression at room temperature along the RD, TD, and ND. As shown, the copper plate exhibits very little plastic anisotropy due to almost random texture (Fig. 2), which is agreement with many other prior works [20, 71-75]. More importantly, the simple Voce law for the threshold stresses for the individual slip systems 
allows the VPSC model to adequately predict the constitutive response. Table 1 lists the final set of Voce parameters for $\mathrm{Cu}$. These parameters are used in the FE-VPSC simulations that follow for $\mathrm{Cu}$.

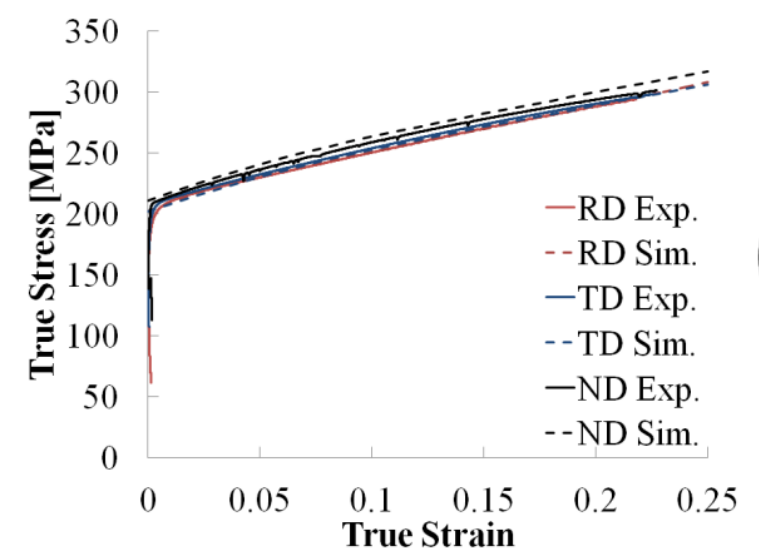

(100)

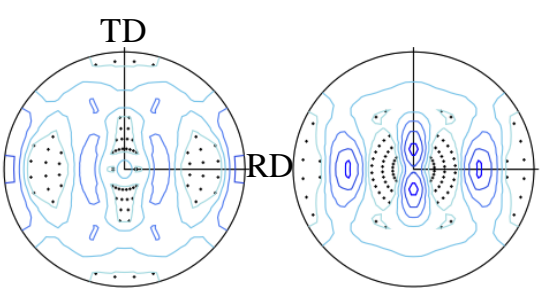

$0.8-1.0-1.2-1.4-1.7-2.0-2.4-$

Figure 2 True stress-true strain response of copper in compression at room temperature along the directions indicated in the plot: measured (solid lines) and predicted (dashed lines). Pole figures measured using X-ray diffraction (XRD) showing initial texture in the annealed copper.

Table 1. Voce hardening law parameters for the constitutive response for $\mathrm{Cu}$.

\begin{tabular}{ccccc}
\hline Material & $\tau_{o o}$ & $\tau_{1}$ & $\theta_{o}$ & $\theta_{1}$ \\
\hline $\mathrm{Cu}$ & 154 & 15 & 180 & 100
\end{tabular}

\subsubsection{Model for $\alpha-U$}

At room temperature, $\alpha-U$ has a base-centered orthorhombic crystal structure with lattice parameters $\mathrm{a}=0.2852 \mathrm{~nm}, \mathrm{~b}=0.5865 \mathrm{~nm}$, and $\mathrm{c}=0.4945 \mathrm{~nm} \mathrm{[16].} \mathrm{It} \mathrm{deforms} \mathrm{by} \mathrm{the} \mathrm{following} \mathrm{slip}$ modes and twinning modes $[15,16,18,76,77]$ : wall slip (010)[100], chimney slip $\frac{1}{2}\{110\}\langle 1 \overline{1} 0\rangle$, floor slip (001)[100], roof slip $\frac{1}{2}\{021\}\langle 1 \overline{1} 2\rangle,\{130\}\langle 3 \overline{1} 0\rangle$ twinning and $\{172\}\langle 3 \overline{1} 2\rangle$ twinning. The prominent deformation twin in $\alpha-U$ is $\{130\}\langle 310\rangle$, and the value of its characteristic twin shear is 0.299 . The $\{172\}\langle 3 \overline{1} 2\rangle$ twin mode and its reciprocal twin $\{112\}\langle 3 \overline{7} 2\rangle$ mode, both with characteristic shears of 0.227 , have also been observed. The twin modes $\{130\}\langle 3 \overline{1} 0\rangle$ and $\{172\}\langle 3 \overline{1} 2\rangle$ reorient the crystal lattice by $69.3^{\circ}$ about [001] and $92.6^{\circ}$ about $\langle\overline{10} 70\rangle$, respectively. These deformation modes are illustrated with respect to the unit cell in [12].

Due to the complex plastic behavior of $\alpha-\mathrm{U}$, we did not use the simple Voce law, but rather a more physically based hardening model. For dislocation slip in this model, the threshold stresses evolve as dislocations are stored in the crystal. Stored dislocations are represented as statistical densities and in the present model this density follows thermally activated rate laws [78-81]. 
These are explicit functions of temperature and strain rate. For twinning, however, the threshold stresses to first form twins evolve with strain but, unlike slip, are not explicit functions of stored dislocations. Stored dislocation density can, however, serve to resist expansion of twin lamellae. More details of this dislocation density (DD) hardening model as it applies to $\alpha-\mathrm{U}$ [13] as well as other metals, like FCC pure $\mathrm{Cu}$ [82], AA6022 [83], and Haynes 25 [84] or BCC Ta [85, 86] and $\mathrm{Nb}$ [87] or HCP Zr [88-90], Be [10], and Mg [4], can be found in prior works. Below we provide an abbreviated review of this model.

The resistance of all slip systems or twin variants within one mode $\alpha$ (or family) in a grain are assumed to exhibit the same resistance. In the case of slip, the resistance is expressed as a sum of a friction stress $\tau_{o}^{\alpha}$, a forest dislocation interaction stress $\tau_{\text {for }}^{\alpha}$, and a dislocation substructure interaction stress $\tau_{s u b}^{\alpha}$, i.e.

$$
\tau_{c}^{\alpha}=\tau_{o}^{\alpha}+\tau_{f o r}^{\alpha}+\tau_{s u b}^{\alpha}
$$

The evolution of $\tau_{\text {for }}^{\alpha}$ and $\tau_{s u b}^{\alpha}$ is governed by the evolution of the forest $\rho_{\text {for }}^{\alpha}$ and substructure $\rho_{s u b}^{\alpha}$ dislocation densities, as explained in detail in [13]. Increases in $\rho_{f o r}^{\alpha}$ and $\rho_{s u b}$ are intended to reflect decreases in the interaction distances between dislocations. Consequently it is expected to lead to increases in the physical barriers to dislocation glide. This phenomenon is captured in the following relationships between $\tau_{\text {for }}^{\alpha}$ and $\tau_{\text {sub }}^{\alpha}$ and $\rho_{\text {for }}^{\alpha}$ and $\rho_{\text {sub }}$ [91, 92]:

$$
\tau_{\text {for }}^{\alpha}=\chi b^{\alpha} \mu^{\alpha} \sqrt{\rho_{\text {for }}^{\alpha}} \text {, and } \tau_{\text {sub }}^{\alpha}=k_{\text {sub }} \mu^{\alpha} b^{\alpha} \sqrt{\rho_{\text {sub }}} \log \left(\frac{1}{b^{\alpha} \sqrt{\rho_{\text {sub }}}}\right)
$$

where the interaction coefficient is $\chi=0.9, \mu^{\alpha}$ the effective shear modulus whose slip system values are taken from [77] for $\alpha-\mathrm{U}$ and from [93] for $\mathrm{Cu}$, and $k_{\text {sub }}=0.086$, a mathematical constant that recovers the Taylor law for low dislocation densities [94]. It is assumed that the evolution of $\rho_{f o r}^{\alpha}$ is determined by a competition between two rates, one for dislocation storage and the other dynamic recovery:

$$
\frac{\partial \rho_{f o r}^{\alpha}}{\partial \gamma^{\alpha}}=\frac{\partial \rho_{g e n, f o r}^{\alpha}}{\partial \gamma^{\alpha}}-\frac{\partial \rho_{r e m, f o r}^{\alpha}}{\partial \gamma^{\alpha}}=k_{1}^{\alpha} \sqrt{\rho_{f o r}^{\alpha}}-k_{2}^{\alpha}(\dot{\varepsilon}, T) \rho_{f o r}^{\alpha}, \quad \Delta \rho_{f o r}^{\alpha}=\frac{\partial \rho_{f o r}^{\alpha}}{\partial \gamma^{\alpha}}\left|\Delta \gamma^{\alpha}\right|,
$$

where $k_{1}^{\alpha}$ is the rate coefficient by statistical trapping of mobile dislocations and $k_{2}^{\alpha}$ is that for dynamic recovery. The ratio of these two coefficients is given by [91]

$$
\frac{k_{2}^{\alpha}}{k_{1}^{\alpha}}=\frac{\chi b^{\alpha}}{g^{\alpha}}\left(1-\frac{k T}{D^{\alpha} b^{3}} \ln \left(\frac{\dot{\varepsilon}}{\dot{\varepsilon}_{o}}\right)\right) \text {. }
$$

In Eq. (9), $k, \dot{\varepsilon}_{o}, g^{\alpha}$, and $D^{\alpha}$ are respectively, Boltzmann's constant, a reference strain rate, an effective activation energy, and a drag stress. In this model, the rate of substructure density is assumed to be coupled to the rate of dynamic recovery of all active dislocations via:

$$
\Delta \rho_{\text {sub }}=\sum_{\alpha} q^{\alpha} b^{\alpha} \sqrt{\rho_{\text {sub }}} \frac{\partial \rho_{\text {rem }, \text { for }}^{\alpha}}{\partial \gamma^{\alpha}}\left|\Delta \gamma^{\alpha}\right|
$$


In Eq. 10, $q$ is a rate coefficient defining the fraction of $\alpha$-type dislocations that do not annihilate but transform eventually into substructure.

The resistance for twin activation accounts for the friction term $\tau_{c}^{\beta}$ and a latent hardening term coupling slip and twin systems using:

$$
\tau_{c}^{\beta}=\tau_{o}^{\beta}+\mu^{\beta} \sum_{\beta} C^{\alpha \beta} b^{\beta} b^{\alpha} \rho_{\text {for }}^{\alpha} .
$$

Here, $\mu^{\beta}, b^{\beta}$ and $C^{\alpha \beta}$ are the elastic shear modulus on the system, the Burgers vector or shear direction of a given system, and the latent hardening matrix, respectively. To model lattice reorientation due to deformation twinning at the grain scale, we employ the composite grain model. In this model, when a grain is selected to form a deformation twin, the grain is partitioned into two representative inclusions, a twin and matrix ellipsoidal inclusion [95]. The crystallographic orientation of the twin corresponds to the one with the highest shear-rate, the socalled predominant twin system (PTS). The short axes of both the matrix and twin inclusions are perpendicular to the twin plane. Once formed, the twin inclusion is allowed to grow, and as it grows, the matrix inclusion shrinks.

The hardening parameters were calibrated in the earlier work [13] by comparing the VPSC simulations with the measured stress strain curves of $\alpha-\mathrm{U}$ in compression and tension. The initial texture was the straight rolling texture of $\alpha-U$ plate. The initial texture, microstructure and the stress-strain response are shown in Fig. 3. The number of grains used to represent this texture in simulation was 8000. These orientations were spreads over an FE element in the FE-VPSC simulations that follow. As mentioned, the $\alpha-U$ plate exhibits significant plastic anisotropy. Due to the strong initial texture and its orientation relationship with respect to the loading cases, in the TD and ND cases, the strain is primarily accommodated by slip, whereas in the RD case, the strain is accommodated by a combination of slip and twinning. Such plastic anisotropy evolution requires polycrystal plasticity models. Thus in what follows, only the VPSC model with deformation twinning and slip with the dislocation density hardening law will be used as the constitutive law in the FE simulations involving $\alpha-U$.

a)

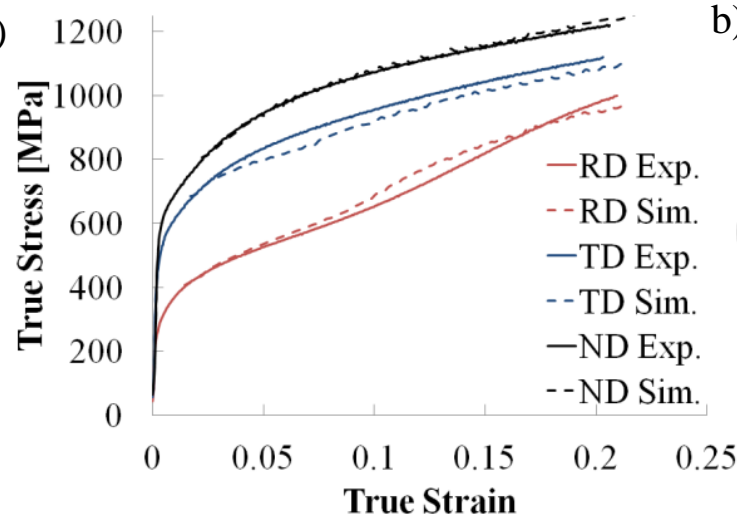

b)

(001)

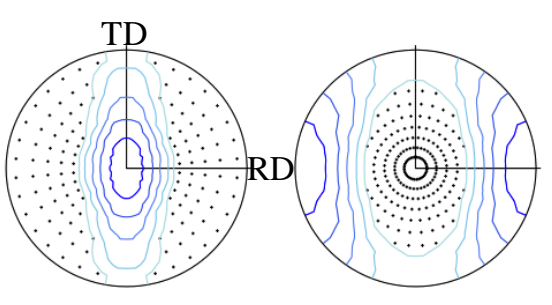

$0.7-1.0-1.5-2.3-3.5-5.3-8.0-$
(100)

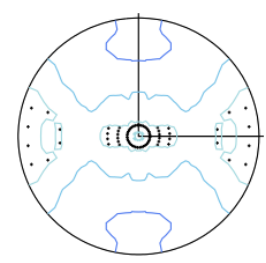


c)

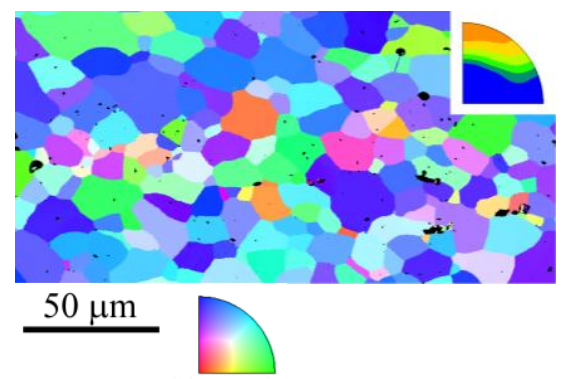

Figure 3 (a) True stress-true strain response of $\alpha$-U in compression at room temperature along the directions indicated in the plot: measured (solid lines) and predicted (dashed lines). (b) Pole figures showing initial texture in the annealed $\alpha$-U. (c) EBSD orientation map showing initial microstructure in the annealed $\alpha$-U. The colors in the map indicate the crystal direction parallel to the rolling direction (unit triangle at the bottom). In the corner of the map we illustrate the inverse pole figure for the rolling direction. The IPF triangle has the crystal reference frame defined as [100] bottom right, [010] top left, and [001] bottom left.

\subsection{FEM implementation}

In this section, we describe the implementation of VPSC as a User MATerial (UMAT) subroutine within ABAQUS-Standard. The total strain increment $(\Delta \boldsymbol{\varepsilon})$ is given as the sum of the elastic $\left(\Delta \boldsymbol{\varepsilon}_{\mathrm{el}}\right)$ and viscoplastic $\left(\Delta \boldsymbol{\varepsilon}_{\mathrm{vp}}\right)$ strains $[26,70]$, i.e.:

$$
\Delta \boldsymbol{\varepsilon}=\Delta \boldsymbol{\varepsilon}_{\mathrm{el}}+\Delta \boldsymbol{\varepsilon}_{\mathrm{vp}}=\boldsymbol{C}^{-1}: \Delta \boldsymbol{\sigma}+\Delta \boldsymbol{\varepsilon}_{\mathrm{vp}}
$$

where $\boldsymbol{C}$ is the elastic stiffness tensor, $\Delta \boldsymbol{\sigma}$ is the Cauchy stress increment, and $\Delta \boldsymbol{\varepsilon}_{\mathrm{vp}}=\Delta \boldsymbol{\varepsilon}_{\mathrm{vp}}(\boldsymbol{\sigma})$ is the viscoplastic strain increment. The latter is computed using the VPSC model. All quantities in Eqn. (12) apply to a given polycrystalline material point.

In its incremental stress update formulation, ABAQUS requires that the macro-scale constitutive model is given in the corrotational frame. Therefore, the model is formulated incrementally using the Jaumann rate from time $t$ to $t+\Delta t$ :

$$
\boldsymbol{\sigma} \Delta \mathrm{t}=\boldsymbol{C}:\left(\Delta \boldsymbol{\varepsilon}-\Delta \boldsymbol{\varepsilon}_{\mathrm{vp}}\right)
$$

The macroscopic applied load is divided into increments and the value of the load increment is determined by time. We obtain equilibrium at each increment by means of the FE analysis. This is done in an iterative fashion using a global nonlinear solver. Once solved at time $t$, the solution for the next time increment requires a tangent stiffness (Jacobian) matrix $C^{\text {tg }}=\partial \Delta \boldsymbol{\sigma} / \partial \Delta \boldsymbol{\varepsilon}$ for each material point in order for the FE scheme to compute an initial guess for the nodal displacements at $t+\Delta t$. In this work, this stiffness matrix is provided by the polycrystal model. The UMAT then uses the predicted strain increments at each material point, $\Delta \boldsymbol{\varepsilon}^{\mathrm{FE}}$, and the stress, $\sigma^{\mathrm{t}}$, and the set of internal state variables corresponding to the previous increment to calculate a new guess for the stress andthe Jacobian at $t+\Delta t$. When equilibrium is achieved by this scheme, the new values for the stresses (at $t+\Delta t$ ), the Jacobian matrix, and the updated set of internal state variables are accepted. The calculation then advances to the next increment. 
The UMAT with the VPSC calculation is based on the minimization procedure described in [26, 70]. In what follows, we summarize this procedure which is used for a given $\Delta \boldsymbol{\varepsilon}^{\mathrm{FE}}$.

The constitutive relationship for the stress at the material point and at $t+\Delta t$ is given by:

$$
\boldsymbol{\sigma}^{\mathrm{t}+\Delta \mathrm{t}}=\boldsymbol{\sigma}^{\mathrm{t}}+\boldsymbol{C}: \Delta \boldsymbol{\varepsilon}_{\mathrm{el}}=\boldsymbol{\sigma}^{\mathrm{t}}+\boldsymbol{C}:\left(\Delta \boldsymbol{\varepsilon}-\Delta \boldsymbol{\varepsilon}_{\mathrm{vp}}\right)
$$

where $\boldsymbol{C}$ is the elastic stiffness tensor of the polycrystal. The common choice for $\boldsymbol{C}$ is the estimate provided from the elastic self-consistent (ELSC) method. VPSC calls on this ELSC calculation for the determination of $\boldsymbol{C}$ at the beginning of each deformation increment. In this way, the elastic modulus of the polycrystalline material element accounts for changes in texture. The elastic constants used for single crystal $\alpha-\mathrm{U}$ are $[77,96]: C_{11}=214.8 \mathrm{GPa}$, $C_{22}=198.6 \mathrm{GPa}, C_{33}=267.1 . \mathrm{GPa}, C_{44}=124.4 \mathrm{GPa}, C_{55}=73.4 \mathrm{GPa}, C_{66}=74.3 \mathrm{GPa}$, and for $\mathrm{Cu}$, they are $C_{11}=168.4 \mathrm{GPa}, C_{12}=121.4 \mathrm{GPa}$, and $C_{44}=75.4 \mathrm{GPa}$.

With both Eq. (14) and the viscoplastic constitutive relation, we get:

$$
\Delta \boldsymbol{\varepsilon}=\boldsymbol{C}^{-1}: \Delta \sigma+\Delta t \dot{\boldsymbol{\varepsilon}}_{v p}^{(p x)}\left(\boldsymbol{\sigma}^{t}+\Delta \boldsymbol{\sigma}\right)
$$

When provided a trial strain increment $\Delta \varepsilon^{\mathrm{FE}}$, we can define the residual at each material point $\mathbf{X}(\Delta \boldsymbol{\sigma})$, as a nonlinear function of the stress increment $\Delta \boldsymbol{\sigma}=\boldsymbol{\sigma}^{\mathrm{t}+\Delta \mathrm{t}}-\boldsymbol{\sigma}^{\mathrm{t}}$ :

$$
\mathbf{X}(\Delta \boldsymbol{\sigma})=\Delta \boldsymbol{\varepsilon}-\Delta \boldsymbol{\varepsilon}^{F E}=\boldsymbol{C}^{-1}: \Delta \boldsymbol{\sigma}+\Delta t \dot{\boldsymbol{\varepsilon}}_{v p}^{(p x)}\left(\boldsymbol{\sigma}^{t}+\Delta \boldsymbol{\sigma}\right)-\Delta \boldsymbol{\varepsilon}^{F E}
$$

Using a Newton-Raphson (NR) scheme to solve the nonlinear system of equations we enforce the condition $\mathbf{X}(\Delta \boldsymbol{\sigma})=0$ (i.e. $\Delta \boldsymbol{\varepsilon}=\Delta \boldsymbol{\varepsilon}^{\mathrm{FE}}$ ), is enforced. This gives the corresponding Jacobian $J_{\mathrm{NR}}$ i.e., :

$$
\begin{aligned}
& \frac{\partial \mathbf{X}(\Delta \boldsymbol{\sigma})}{\partial(\Delta \boldsymbol{\sigma})}=\boldsymbol{J}_{N R}(\Delta \boldsymbol{\sigma})=\boldsymbol{C}^{t g}= \\
& \quad=\boldsymbol{C}^{-1}+\Delta t \frac{\partial \dot{\boldsymbol{\varepsilon}}_{v p}^{(p x)}}{\partial(\Delta \boldsymbol{\sigma})}\left(\boldsymbol{\sigma}^{t}+\Delta \boldsymbol{\sigma} ; \beta_{i}^{t}\right)=\boldsymbol{C}^{-1}+\Delta t \boldsymbol{M}^{(p x)}\left(\boldsymbol{\sigma}^{t}+\Delta \boldsymbol{\sigma}\right)
\end{aligned}
$$

When provided a guess for the stress increment $\Delta \boldsymbol{\sigma}^{\mathrm{k}-1}$, we can obtain the new guess by:

$$
\Delta \boldsymbol{\sigma}^{\mathrm{k}}=\Delta \boldsymbol{\sigma}^{\mathrm{k}-1}-\boldsymbol{J}_{\mathrm{NR}}^{-1}\left(\Delta \boldsymbol{\sigma}^{\mathrm{k}-1}\right): \mathbf{X}\left(\Delta \boldsymbol{\sigma}^{\mathrm{k}-1}\right)
$$

The above approach gives a closed expression for the FE Jacobian as a function of the FE time increment, the elastic stiffness of the aggregate, and the viscoplastic tangent moduli (calculated as part of the VPSC algorithm). Using this expression reduces substantially computational cost since the elasto-viscoplastic tangent stiffness tensor and the polycrystal stress can be calculated within the same calculation loop. In addition, the FE Jacobian allows for quadratic convergence of the macroscopic nonlinear equations.

In the calculations that follow, we begin with $\mathrm{Cu}$ and then follow with $\alpha$-U. For $\mathrm{Cu}$, due to its relatively simple plastic behavior, many options could be considered appropriate. We employed either VPSC or isotropic J2 plasticity at all FE integration points. The former one is more computationally intensive and the latter more computationally efficient. To strike a balance between accuracy and computational effort, we additionally considered two more models, either a combination of the FE-VPSC model (used in the region around the hole) and the isotropic J2 
plasticity (used everywhere else) or a combination of the FE-VPSC model (used in the region around the hole) and the isotropic elasticity (used everywhere else). In these cases, the hole region consisted of those integration points within the insert defined in Fig. 1. For $\alpha-U$, we considered only the FE-VPSC with the DD hardening law at all integration points. This decision was based on the results of the $\mathrm{Cu}$, as we will discuss shortly, and the fact that $\alpha$ - $U$ is highly plastically anisotropic.

\section{Results}

Figure 1 shows the plate assembly used in this work. For all simulations, the FE model consisted of linear C3D8 (8 nodes, and 8 integration points) elements. Symmetry was utilized to produce a quarter model for computational efficiency and the quarter model consisted, after a mesh convergence study, of approximately 600 elements.

\subsection{Applications of the FE-VPSC to $C u$}

In this section, we use the multiscale model to simulate the pulling apart of two $\mathrm{Cu}$ plates joined by a pin. Figure 4 shows the axial strain contours generated in the $\mathrm{Cu}$ plate assembly at $0.1 \mathrm{~mm}$ applied displacement. Calculations are shown for the four models mentioned above. Cases involving VPSC used $\mathrm{N}=2916$ grains for the representative polycrystal. As shown, all models give nearly the same strain distribution with the exception of the model that uses the fine-scale VPSC model in the region around the hole and isotropic J2 plasticity model elsewhere. The former set of models finds that the highest and lowest strains develop in the local region at the hole/pin interface. The model that combines VPSC with J2 plasticity, in contrast, predicts that the higher strain regions develop away from the hole/pin interface.

Due to the differences between the models with different constitutive laws, we sought validation with experimental measurement. To this end, we elected to assess the capability of each model by comparing with a DIC analysis of a mock set up. The mock set up of the pin-plate assembly was constructed using OFHC copper for the plate and hardened steel for the pins. In-situ displacement tests along the plate RD direction were conducted while DIC data was collected. Correlated Solutions DIC software was used to analyze 2D displacements around the hole. For the analysis, we selected an area of interest to be a square section around the pin with the pin area subtracted. Based on the size of the speckles in the pattern, a subset size of 65 by 65 pixels was set up with a step size of 10 pixels. The correlation was completed using the cubic B-spline interpolation, affine transformation subset shape function, and normalized sum of squared differences.

For the FE simulation, the applied strain rate and boundary conditions equivalent to the experiment, i.e., $0.001 \mathrm{~s}^{-1}$. The boundary conditions for this test are displacements imposed along the 2-direction and stress-free boundary conditions on the lateral faces. The pin was modeled as a rigid body. Again, three constitutive laws for $\mathrm{Cu}$ were used: isotropic $\mathrm{J} 2$ plasticity (labeled 'isotropic'), FE-VPSC considering 2916 grains at each FE integration point (labeled 'FE+VPSC'), and a combination of the FE-VPSC model (used in the region around the hole) and isotropic $\mathrm{J} 2$ plasticity (used everywhere else). 
Figure 5 shows the comparison between the DIC measurements and the FE simulation of the axial and in-plane shear distributions calculated from the three models. As shown, the FE models employing VPSC only or $\mathbf{J} 2$ plasticity theory only capture the heterogeneous strain distributions in the hole region. The contours predicted by J2 and FE-VPSC are similar. This is expected because $\mathrm{Cu}$ has a high symmetry FCC crystal structure that does not exhibit substantial anisotropy in material properties for textures with intensity less than 5 MRD (multiples of random distribution) and can be approximated using J2 plasticity [97]. However because the FEVPSC model captures texture evolution, some degree of material anisotropy may develop and, therefore, some discrepancy in the contour plots predicted by $\mathrm{J} 2$ and FE-VPSC can be expected. One of the benefits of the FE-VPSC model is the prediction of texture evolution and the associated development of anisotropy in the material response. Between these two more successful cases, we find that the FE+VPSC model better predicts the intensity and extent of the strains in this region.

Perhaps it is not surprising that the FE model with the fine-scale VPSC calculation at every integration point produces the best agreement with the experiment. However, we do find it interesting that the combination of the FE-VPSC model (used in the region around the hole) and isotropic J2 plasticity (used everywhere else), which served as a compromise in computation time, produces the most disagreement. Evidently, combining multiple constitutive laws undermines the predictive capability and it is better in this case to use the same constitutive law for all integration points. This result suggests that a sharp transition from one to another computational subdomain, in our case from the rest of the plate to the insert, requires a sandwich domain that would acts as an interface region to regularize any kinematic incompatibilities. Alternative continuity conditions that would facilitate the 'smooth' transition across the boundaries between the constitutive laws have not been explored.

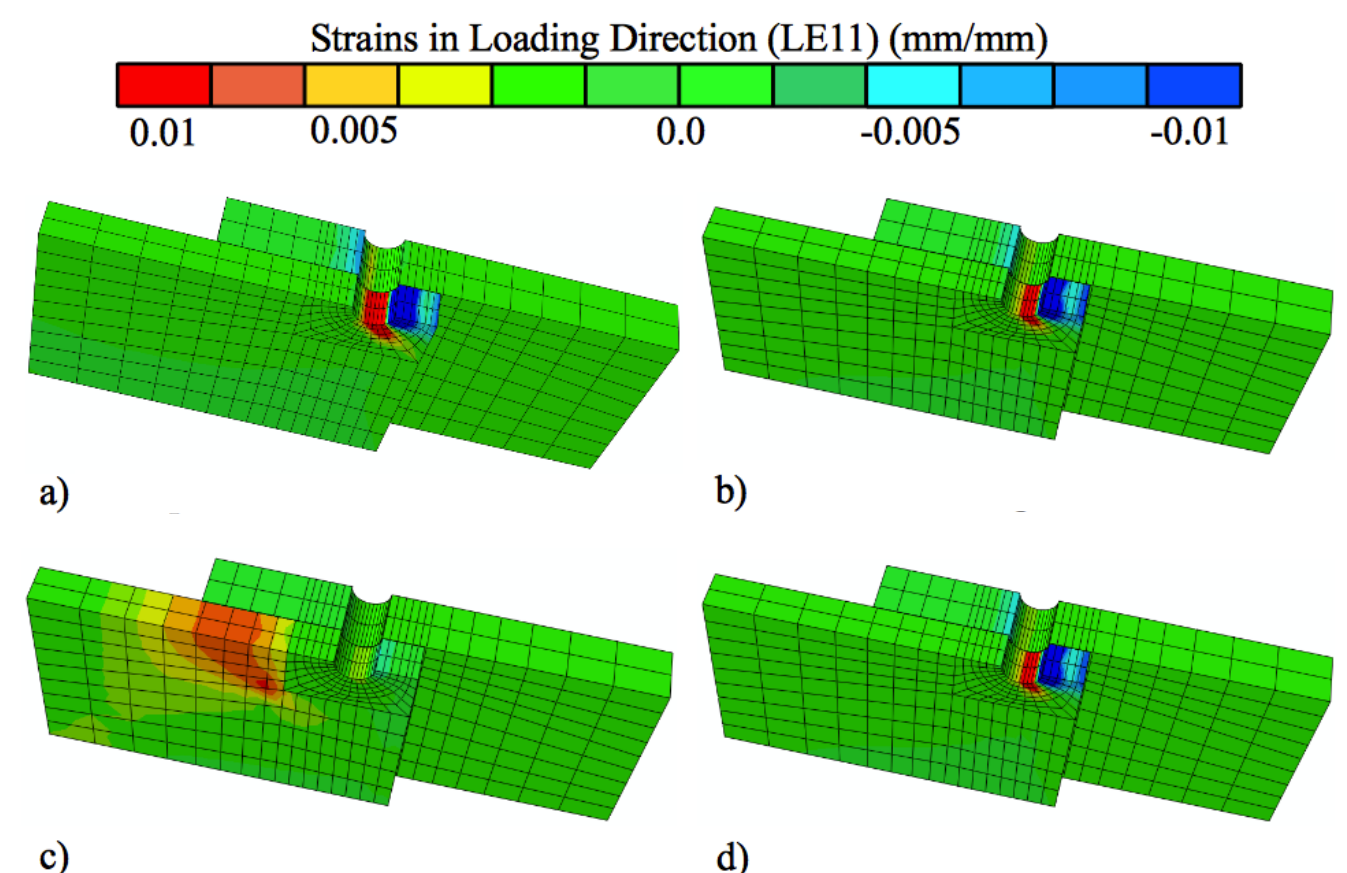

c)

d) 
Figure 4 Axial strain contours predicted by different constitutive laws at $0.1 \mathrm{~mm}$ of applied displacement: (a) isotropic J2 plasticity, (b) FE-VPSC considering 2916 grains at each FE integration point, (c) a combination of the FE-VPSC model (used in the region around the hole) and the isotropic $\mathrm{J} 2$ plasticity (used everywhere else), and (d) a combination of the FE-VPSC model (used in the region around the hole) and the isotropic elasticity (used everywhere else).

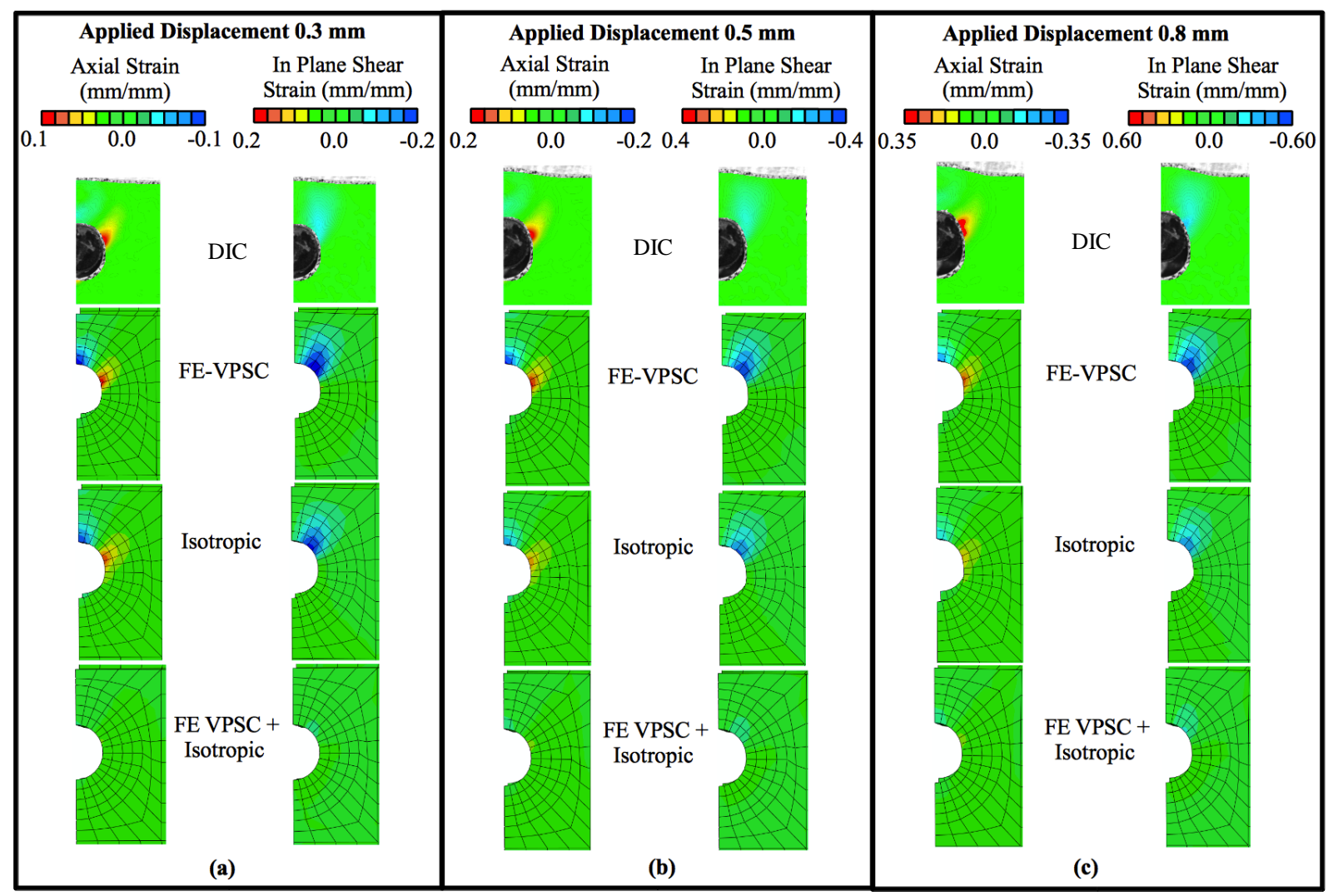

Figure 5 Comparison of axial and shear strain contours measured by digital image correlation against predicted by different constitutive laws at three stages of applied displacement indicated in the figure.

For a simple material like $\mathrm{Cu}$, we have explored several options for its constitutive law. Thus far, we find that the FE calculation using the fine-scale VPSC constitutive model at each integration point performs very well and the best among the cases tested here. An important consideration, however, when choosing this type of calculation is the computational time required. Moreover, for VPSC, an additional option is the number of grain orientations $\mathrm{N}$ used to represent the polycrystal at each integration point. Figure 7 compares the computation times for the same simulation but different $\mathrm{N}$ for VPSC-only cases, combinations of VPSC $+\mathrm{J} 2$ theory, and $\mathrm{J} 2$ theory-only. Computation times are shown for a total displacement of $3 \mathrm{~mm}$. For the comparison of the VPSC-only cases with different $\mathrm{N}$, we ensured that the stress-strain curves obtained for all values of $\mathrm{N}$ in the range from 2916 to 500 grains were the same (see Fig. 6) as well as the strain contours in the plate simulation. Note that any localized grain scale response or misorientation distributions predicted by different numbers of grains will likely not correspond to a dependence 
on the number of grains. As shown in Fig. 7, not surprisingly, the VPSC-only calculation with 2916 grains is the most computationally intensive, followed by the VPSC $+\mathrm{J} 2$ theory combination again with $\mathrm{N}=2916$ grains for the VPSC portion, and then $\mathrm{J} 2$ theory-only case, the most computationally efficient. The difference between the FE-VPSC 2916 grains Isotropic 1 and 2 is in the size of the insert. While the insert size is $10 \mathrm{~mm}$ in the former case, it is twice as big in the later case. Notably, the VPSC-only simulation involving $\mathrm{N}=500$ grains is remarkably efficient, giving a much lower computation time compared to the VPSC-only calculation and the VPSC $+\mathrm{J} 2$ theory combination with $\mathrm{N}=2916$ grains. To summarize, to strike a balance between accuracy and computational efficiency, the use of VPSC with the minimum possible number of grains is recommended.

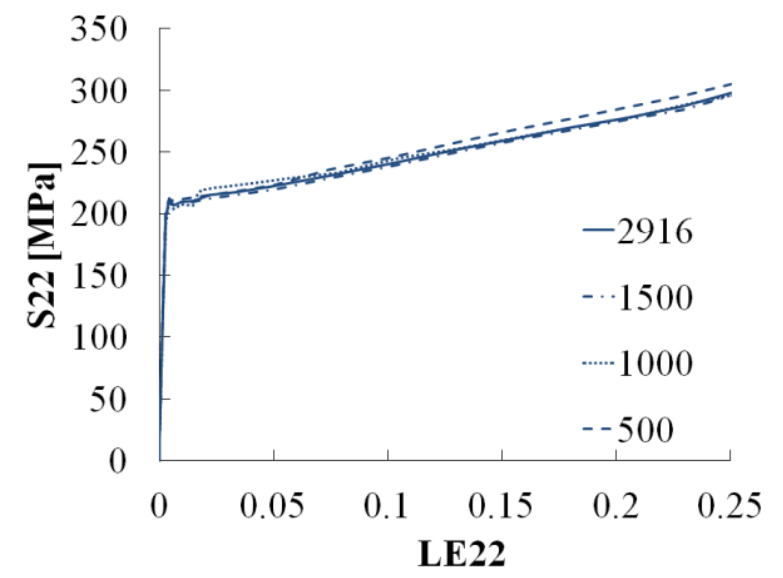

Figure 6 Stress-strain curves obtained by the FE-VPSC model considering different numbers of grains indicated in the figure. The FE model consisted of single linear element C3D8 (8 nodes, and 8 integration points) with displacements imposed along the 2-direction and stress-free boundary conditions on the lateral faces.

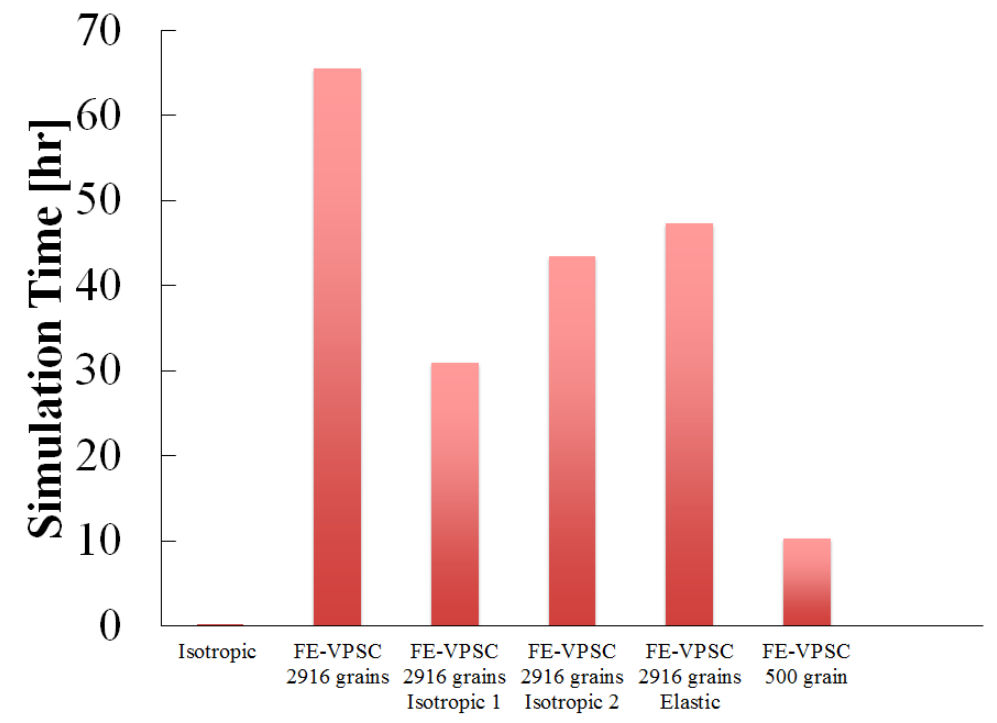

Figure 7 Comparison of elapsed simulation times corresponding to the simulations using the constitutive laws or different combinations of constitutive laws indicated in the figure. 
Another critical factor is the opening displacements of the hole, which is also sensitive to the choice of constitutive law. With the FE model employed here, we can also estimate the evolution of the opening on the hole with applied deformation and compare it with the DIC measurement. Here the opening of hole is defined as displacement of the contact points between the pin and the outer plates at the top of the hole on the symmetry plane parallel with the loading direction. These contact points experience the most displacement during the simulation/experiment of the joint as they move upward with the applied displacement. The hole changes its shape from a perfect circle to a distorted ellipse with deformation. The hole-opening is defined along the major axis of this ellipse. Figure 8 compares the calculated hole-opening using different constitutive laws and the measured data. Specifically, the plot compares how much displacement has been applied on the inner plate versus how much the hole has opened at the points of pin contact. Anisotropic material models will vary in this response depending on the material orientation compared to isotropic materials. As before, we see that the simulations using a combination of VPSC and J2 plasticity theory are the outlier cases, producing a much lower hole opening than the VPSC-only or J2 plasticiy theory-only simulations. The plot also contains a comparison of two FE-VPSC calculations using either $\mathrm{N}=2916$ or 500 grains per integration point. Interestingly, we do not see an influence on hole opening of the number of grains chosen for the VPSC calculation. This result is likely a consequence of the fact that the stress-strain response in Fig. 6 is not significantly different between the FE-VPSC-2916 and FE-VPSC-500 models. While the early potion of the curve is best predicted by the FE-VPSC only model likely to be due to this model best capturing the elasto-plastic transition, the later portion of the curve appear to be best predicted by the isotropic $\mathrm{J} 2$ model. However, the measurements should be considered as the lower bound estimate because of the small deformation of the pin while the simulations are upper bound since the pin is rigid.

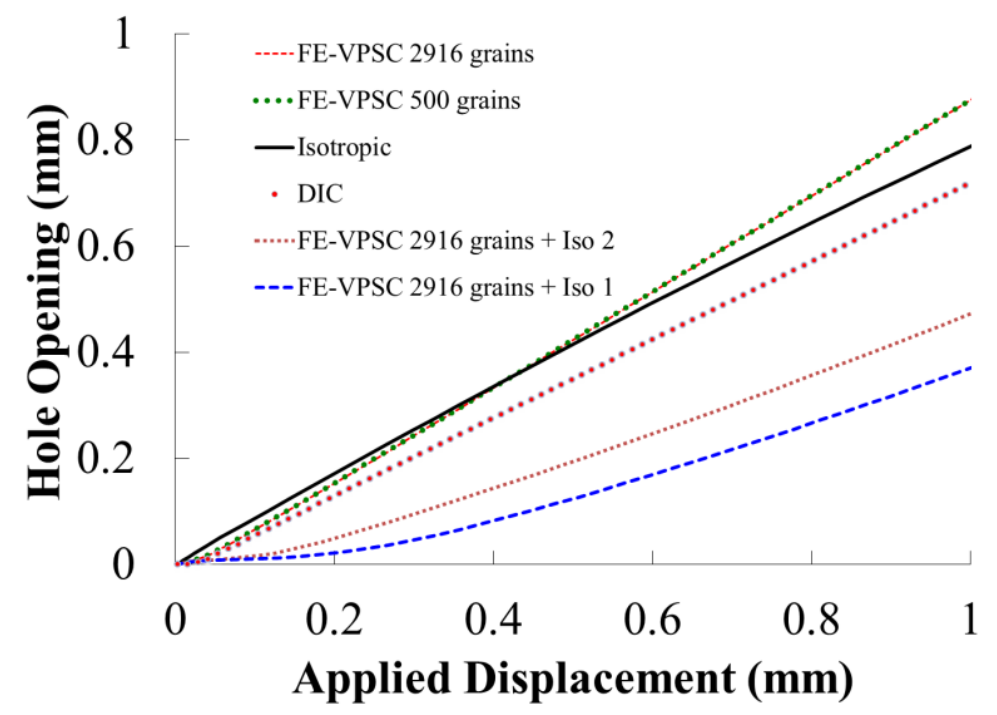

Figure 8 Measured and predicted opening of the hole in the $\mathrm{Cu}$ plate as a function of applied displacement. 


\subsection{Applications of the FE-VPSC to $\alpha-U$}

In this section, we repeat the deformation simulation to study the pulling apart of two plates made of $\alpha-U$ joined by a rigid steel pin. All integration points use the VPSC model to supply the constitutive response. Before the load is applied, the polycrystal model at every point is assigned the same initial texture, which is taken directly from the measurement of the rolled sheet. The VPSC polycrystals at FE integration points are represented by 1000 grains.
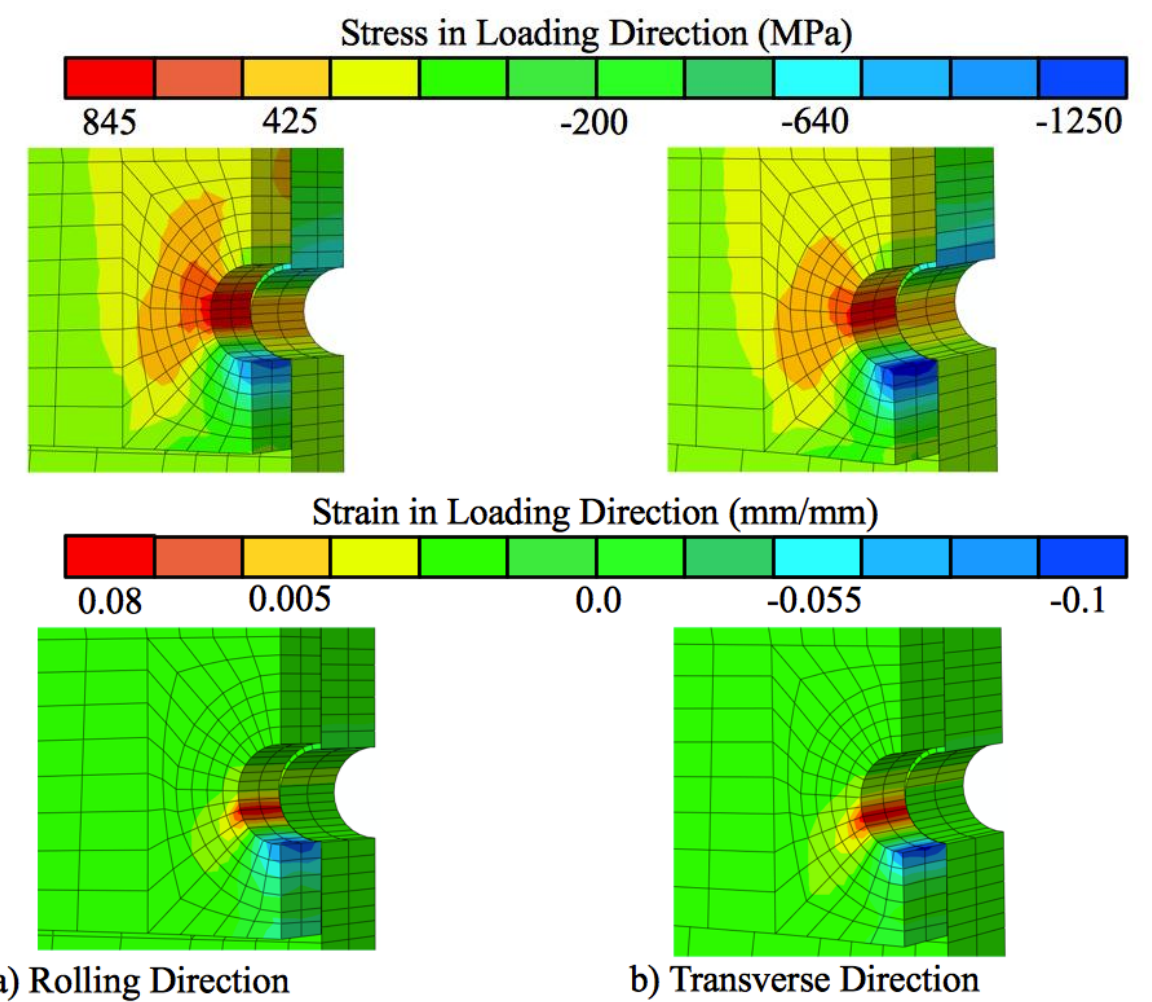

Figure 9 Axial stress and strain contours predicted by the FE-VPSC model in two different loading directions.

We first apply the model to study the deformation state in the plate as a load is applied in one of two different loading directions, either the rolling direction (RD) or the transverse direction (TD). Figure 9 shows the axial stress and strain contours in (a) the RD and (b) the TD. We observe that the strain concentrates in the hole region where the plate and rigid pin intersect. We also see that the extent and magnitude of the strain and stress concentration regions depends on the loading direction. This difference is a consequence of the plastic anisotropy of the plate due to 1) the strong initial texture of the plate and 2) the multiple slip and twin systems activated in each grain of each polycrystal at each integration point.

In order to better illustrate the plastic anisotropy seen in Fig. 9, we extracted the stress-strain states at the top of the hole (the state of compression) and 90 degrees apart (the state of tension). 
At this location, the loading on the hole is the closest to tensile. Instead of extracting the data from the location where the total tensile strain exhibits the hotspot, we used the 90 degree location where the contour values are about 0.01 because the loading at the hot spot is a mixture of strain components due to the curvature of the hole. Using the two locations 90 degrees apart also makes the most sense from the perspective of being consistent between the two simulations. Figure 10 plots the predicted constitutive response of $\alpha-U$ for both the RD and the TD loading cases from Fig. 9. First we see a pronounced tension-compression asymmetry. In the TD loading, the compression response has a higher flow stress than the tension response. In the RD loading, the tension-compression asymmetry is less severe than in TD and in contrast, tension has the higher flow stress. Clearly, the J2 isotropic plasticity model will not be able to capture such complex material behavior.

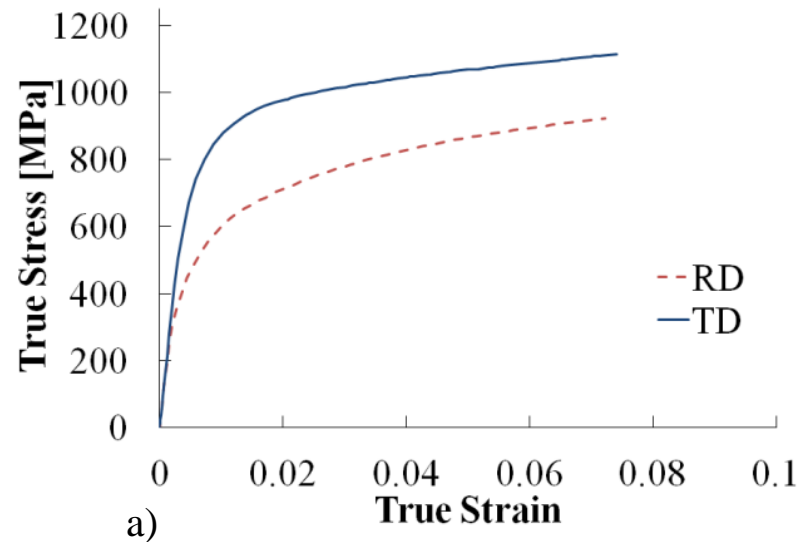

a)

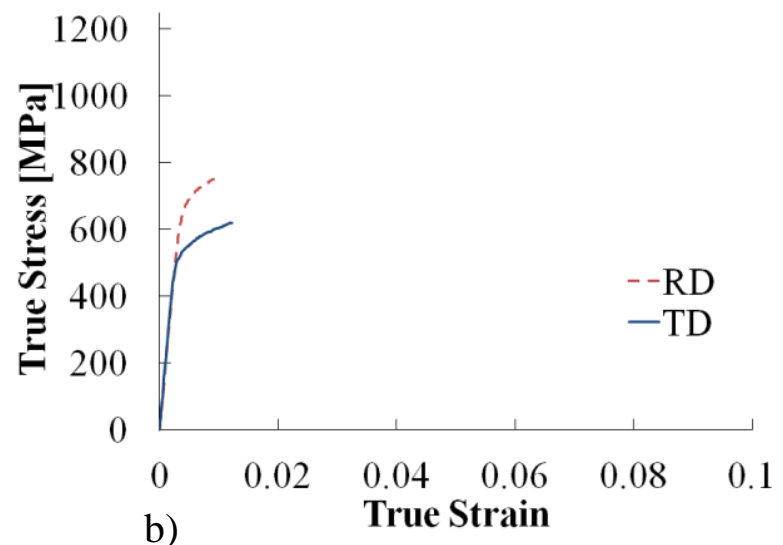

b)

Figure 10 Predicted anisotropic stress-strain response in (a) compression and (b) tension locations around the hole in Fig. 9 during loading along the two directions of the $\alpha$-U plate.

\section{Conclusions}

In this work, we build a multi-scale finite element (FE) model to simulate the deformation of a pinned-joint plate assembly. With this model, simulations are performed to calculate the deformation in the vicinity of the hole in the plate where it meets a rigid pin. Two plate materials are considered: copper and $\alpha-U$. For copper, digital image correlation (DIC) tests are carried out during loading of the plate/pin assembly to characterize the strain distributions in this same critical area. In the FE calculation, we considered different constitutive laws, either a fine-scale polycrystal plasticity or J2 flow theory or both. We show that calculations using the fine-scale polycrystal plasticity constitutive law successfully captures the DIC strain fields in the hole region at different plate displacements. Use of the more computationally efficient $\mathrm{J} 2$ plasticity model also produces reasonable results in comparison with the measurements and the fine-scale constitutive law. Surprisingly, use of the fine-scale constitutive laws in the region surrounding the hole and continuum $\mathrm{J} 2$ theory elsewhere gives the worst agreement and non-conservative estimates for the hole opening with applied displacement. We also repeated this simulation using $\alpha-U$ as the plate material. In this case, use of the fine-scale polycrystal simulation is important considering the highly anisotropic plastic response of this complicated material. The analysis 
predicts that in $\alpha-\mathrm{U}$ plate, the localized deformation in the hole region is highly dependent on the direction of displacement.

\section{Acknowledgements}

This work was supported by the Los Alamos National Laboratory Directed Research and Development (LDRD) project 20140630ER. Los Alamos National Laboratory is operated by Los Alamos National Security LLC under DOE Contract DE-AC52-06NA25396. M. K. gratefully acknowledges the Seaborg Institute for partial financial support under a Post-Doctoral Fellowship through the LANL LDRD Program.

\section{References}

[1] R.L. Norton, Machine Design: An Integrated Approach, Prentice Hall, 2013.

[2] J.E. Shigley, Shigley's mechanical engineering design, McGraw-Hill Education, 2011.

[3] M. Lentz, M. Klaus, I.J. Beyerlein, M. Zecevic, W. Reimers, M. Knezevic, In situ X-ray diffraction and crystal plasticity modeling of the deformation behavior of extruded $\mathrm{Mg}-\mathrm{Li}-(\mathrm{Al})$ alloys: An uncommon tension-compression asymmetry, Acta Materialia, 86 (2015) 254-268.

[4] M. Lentz, M. Klaus, M. Wagner, C. Fahrenson, I.J. Beyerlein, M. Zecevic, W. Reimers, M. Knezevic, Effect of age hardening on the deformation behavior of an Mg-Y-Nd alloy: In-situ Xray diffraction and crystal plasticity modeling, Materials Science and Engineering: A, 628 (2015) 396-409.

[5] M. Knezevic, A. Levinson, R. Harris, R.K. Mishra, R.D. Doherty, S.R. Kalidindi, Deformation twinning in AZ31: Influence on strain hardening and texture evolution, Acta Materialia, 58 (2010) 6230-6242.

[6] B.S. Fromm, B.L. Adams, S. Ahmadi, M. Knezevic, Grain size and orientation distributions: Application to yielding of $\alpha$-titanium, Acta Materialia, 57 (2009) 2339-2348.

[7] A.A. Salem, S.R. Kalidindi, R.D. Doherty, Strain hardening of titanium: role of deformation twinning, Acta Materialia, 51 (2003) 4225-4237.

[8] M. Knezevic, I.J. Beyerlein, T. Nizolek, N.A. Mara, T.M. Pollock, Anomalous Basal Slip Activity in Zirconium under High-strain Deformation, Materials Research Letters, 1 (2013) 133140.

[9] M. Knezevic, M. Zecevic, I.J. Beyerlein, J.F. Bingert, R.J. McCabe, Strain rate and temperature effects on the selection of primary and secondary slip and twinning systems in HCP $\mathrm{Zr}$, Acta Materialia, 88 (2015) 55-73.

[10] M. Knezevic, I.J. Beyerlein, D.W. Brown, T.A. Sisneros, C.N. Tomé, A polycrystal plasticity model for predicting mechanical response and texture evolution during strain-path changes: Application to beryllium, International Journal of Plasticity, 49 (2013) 185-198.

[11] M. Zecevic, M. Knezevic, I.J. Beyerlein, C.N. Tomé, An elasto-plastic self-consistent model with hardening based on dislocation density, twinning and de-twinning: Application to strain path changes in HCP metals, Materials Science and Engineering: A, 638 (2015) 262-274.

[12] R.J. McCabe, L. Capolungo, P.E. Marshall, C.M. Cady, C.N. Tomé, Deformation of wrought uranium: Experiments and modeling, Acta Materialia, 58 (2010) 5447-5459.

[13] M. Knezevic, L. Capolungo, C.N. Tomé, R.A. Lebensohn, D.J. Alexander, B. Mihaila, R.J. McCabe, Anisotropic stress-strain response and microstructure evolution of textured $\alpha$-uranium, Acta Materialia, 60 (2012) 702-715. 
[14] R.J. McCabe, A.W. Richards, D.R. Coughlin, K.D. Clarke, I.J. Beyerlein, M. Knezevic, Microstructure effects on the recrystallization of low-symmetry alpha-uranium, Journal of Nuclear Materials, 465 (2015) 189-195.

[15] R.W. Cahn, Twinning and slip in $\alpha$-uranium, Acta Crystallographica, 4 (1951) 470.

[16] R.W. Cahn, Plastic deformation of alpha-uranium; twinning and slip, Acta Metallurgica, 1 (1953) 49-70.

[17] M.H. Yoo, Slip modes of alpha uranium, Journal of Nuclear Materials, 26 (1968) 307-318.

[18] J.S. Daniel, B. Lesage, P. Lacombe, The influence of temperature on slip and twinning in uranium, Acta Metallurgica, 19 (1971) 163-173.

[19] S.R. Kalidindi, L. Anand, An approximate procedure for predicting the evolution of crystallographic texture in bulk deformation processing of fcc metals, International Journal of Mechanical Sciences, 34 (1992) 309-329.

[20] M. Knezevic, S.R. Kalidindi, D. Fullwood, Computationally efficient database and spectral interpolation for fully plastic Taylor-type crystal plasticity calculations of face-centered cubic polycrystals, International Journal of Plasticity, 24 (2008) 1264-1276.

[21] I.J. Beyerlein, C.N. Tomé, Modeling transients in the mechanical response of copper due to strain path changes, International Journal of Plasticity, 23 (2007) 640-664.

[22] C.A. Calhoun, E. Garlea, R.P. Mulay, T.A. Sisneros, S.R. Agnew, Investigation of the effect of thermal residual stresses on deformation of $\alpha$-uranium through neutron diffraction measurements and crystal plasticity modeling, Acta Materialia, 85 (2015) 168-179.

[23] R. Field, R. McCabe, D. Alexander, D. Teter, Deformation twinning and twinning related fracture in coarse-grained $\alpha$-uranium, Journal of Nuclear Materials, 392 (2009) 105-113.

[24] A.J. Beaudoin, K.K. Mathur, P.R. Dawson, G.C. Johnson, Three-dimensional deformation process simulation with explicit use of polycrystal plasticity models, International Journal of Plasticity, 9 (1993) 833-860.

[25] K. Inal, R.K. Mishra, O. Cazacu, Forming simulation of aluminum sheets using an anisotropic yield function coupled with crystal plasticity theory, International Journal of Solids and Structures, 47 (2010) 2223-2233.

[26] J. Segurado, R.A. Lebensohn, J. Llorca, C.N. Tomé, Multiscale modeling of plasticity based on embedding the viscoplastic self-consistent formulation in implicit finite elements, International Journal of Plasticity, 28 (2012) 124-140.

[27] M. Knezevic, R.A. Lebensohn, O. Cazacu, B. Revil-Baudard, G. Proust, S.C. Vogel, M.E. Nixon, Modeling bending of $\alpha$-titanium with embedded polycrystal plasticity in implicit finite elements, Materials Science and Engineering: A, 564 (2013) 116-126.

[28] K.W. Neale, Use of crystal plasticity in metal forming simulations, International Journal of Mechanical Sciences, 35 (1993) 1053-1063.

[29] E. Nakamachi, T. Honda, H. Kuramae, Y. Morita, T. Ohata, H. Morimoto, Two-scale finite element analyses for bendability and springback evaluation based on crystallographic homogenization method, International Journal of Mechanical Sciences, 80 (2014) 109-121.

[30] E. Nakamachi, T. Honda, H. Kuramae, Y. Morita, H. Morimoto, Process metallurgy analyses to design a high-bendability and high-springback property sheet by using two-scale finite element method, International Journal of Mechanical Sciences, 87 (2014) 89-101.

[31] S. Balasubramanian, L. Anand, Single crystal and polycrystal elasto-viscoplasticity: Application to earing in cup drawing of F.C.C. materials, Comput Mech, 17 (1996) 209-225.

[32] P.J. Maudlin, S.K. Schiferl, Computational anisotropic plasticity for high-rate forming applications, Computer Methods in Applied Mechanics and Engineering, 131 (1996) 1-30. 
[33] D. Raabe, F. Roters, Using texture components in crystal plasticity finite element simulations, International Journal of Plasticity, 20 (2004) 339-361.

[34] C.L. Xie, E. Nakamachi, Investigations of the formability of BCC steel sheets by using crystalline plasticity finite element analysis, Materials \& Design, 23 (2002) 59-68.

[35] A.J. Beaudoin, P.R. Dawson, K.K. Mathur, U.F. Kocks, D.A. Korzekwa, Application of polycrystal plasticity to sheet forming, Computer Methods in Applied Mechanics and Engineering, 117 (1994) 49-70.

[36] A. Kumar, P.R. Dawson, Polycrystal plasticity modeling of bulk forming with finite elements over orientation space, Comput Mech, 17 (1995) 10-25.

[37] M. Knezevic, M. Jahedi, Y.P. Korkolis, I.J. Beyerlein, Material-based design of the extrusion of bimetallic tubes, Computational Materials Science, 95 (2014) 63-73.

[38] M. Zecevic, R.J. McCabe, M. Knezevic, A new implementation of the spectral crystal plasticity framework in implicit finite elements, Mechanics of Materials, 84 (2015) 114-126.

[39] M. Zecevic, R.J. McCabe, M. Knezevic, Spectral database solutions to elasto-viscoplasticity within finite elements: Application to a cobalt-based FCC superalloy, International Journal of Plasticity, 70 (2015) 151-165.

[40] D.E. Green, K.W. Neale, S.R. MacEwen, A. Makinde, R. Perrin, Experimental investigation of the biaxial behaviour of an aluminum sheet, International Journal of Plasticity, 20 (2004) 1677-1706.

[41] C.P. Kohar, M. Mohammadi, R.K. Mishra, K. Inal, Effects of elastic-plastic behaviour on the axial crush response of square tubes, Thin-Walled Structures, 93 (2015) 64-87.

[42] M. Kraska, M. Doig, D. Tikhomirov, D. Raabe, F. Roters, Virtual material testing for stamping simulations based on polycrystal plasticity, Computational materials science, 46 (2009) 383-392.

[43] P. Van Houtte, S. Li, O. Engler, Taylor - Type Homogenization Methods for Texture and Anisotropy, Continuum Scale Simulation of Engineering Materials: FundamentalsMicrostructures-Process Applications, (2004) 459-472.

[44] Mathur, K. K., P.R. Dawson, On modelling the development of crystallographic texture in bulk forming processes, International Journal of Plasticity, 5 (1989) 67-94.

[45] G.B. Sarma, P.R. Dawson, Texture predictions using a polycrystal plasticity model incorporating neighbor interactions, International Journal of Plasticity, 12 (1996) 1023-1054.

[46] N.R. Barton, J. Knap, A. Arsenlis, R. Becker, R.D. Hornung, D.R. Jefferson, Embedded polycrystal plasticity and adaptive sampling, International Journal of Plasticity, 24 (2008) 242266.

[47] H.F. Al-Harbi, M. Knezevic, S.R. Kalidindi, Spectral approaches for the fast computation of yield surfaces and first-order plastic property closures for polycrystalline materials with cubictriclinic textures, CMC: Computers, Materials, \& Continua, 15 (2010) 153-172.

[48] M. Knezevic, H.F. Al-Harbi, S.R. Kalidindi, Crystal plasticity simulations using discrete Fourier transforms, Acta Materialia, 57 (2009) 1777-1784.

[49] M. Knezevic, S.R. Kalidindi, Fast computation of first-order elastic-plastic closures for polycrystalline cubic-orthorhombic microstructures, Computational Materials Science, 39 (2007) 643-648.

[50] M. Knezevic, S.R. Kalidindi, R.K. Mishra, Delineation of first-order closures for plastic properties requiring explicit consideration of strain hardening and crystallographic texture evolution, International Journal of Plasticity, 24 (2008) 327-342. 
[51] S.R. Kalidindi, H.K. Duvvuru, M. Knezevic, Spectral calibration of crystal plasticity models, Acta Materialia, 54 (2006) 1795-1804.

[52] S.R. Kalidindi, M. Knezevic, S. Niezgoda, J. Shaffer, Representation of the orientation distribution function and computation of first-order elastic properties closures using discrete Fourier transforms, Acta Materialia, 57 (2009) 3916-3923.

[53] N. Landry, M. Knezevic, Delineation of First-Order Elastic Property Closures for Hexagonal Metals Using Fast Fourier Transforms, Materials, 8 (2015) 6326-6345.

[54] X. Wu, G. Proust, M. Knezevic, S.R. Kalidindi, Elastic-plastic property closures for hexagonal close-packed polycrystalline metals using first-order bounding theories, Acta Materialia, 55 (2007) 2729-2737.

[55] M. Knezevic, D.J. Savage, A high-performance computational framework for fast crystal plasticity simulations, Computational Materials Science, 83 (2014) 101-106.

[56] B. Mihaila, M. Knezevic, A. Cardenas, Three orders of magnitude improved efficiency with high - performance spectral crystal plasticity on GPU platforms, International Journal for Numerical Methods in Engineering, 97 (2014) 785-798.

[57] D.J. Savage, M. Knezevic, Computer implementations of iterative and non-iterative crystal plasticity solvers on high performance graphics hardware, Computational Mechanics 56 (2015) 677-690.

[58] M. Knezevic, N.W. Landry, Procedures for reducing large datasets of crystal orientations using generalized spherical harmonics, Mechanics of Materials, 88 (2015) 73-86.

[59] T. Fast, M. Knezevic, S.R. Kalidindi, Application of microstructure sensitive design to structural components produced from hexagonal polycrystalline metals, Computational Materials Science, 43 (2008) 374-383.

[60] E. Nakamachi, T. Honda, H. Kuramae, Y. Morita, T. Ohata, H. Morimoto, Two-scale finite element analyses for bendability and springback evaluation based on crystallographic homogenization method, International Journal of Mechanical Sciences, 80 (2014) 109-121.

[61] J.C. Simo, R.L. Taylor, Consistent tangent operators for rate-independent elastoplasticity, Computer Methods in Applied Mechanics and Engineering, 48 (1985) 101-118.

[62] R.A. Lebensohn, C.N. Tomé, A self-consistent anisotropic approach for the simulation of plastic deformation and texture development of polycrystals: Application to zirconium alloys, Acta Metallurgica et Materialia, 41 (1993) 2611-2624.

[63] R.A. Lebensohn, C.N. Tomé, P.P. Castaneda, Self-consistent modelling of the mechanical behaviour of viscoplastic polycrystals incorporating intragranular field fluctuations, Philosophical Magazine, 87 (2007) 4287-4322.

[64] Correlated Solutions, Inc., 2013.

[65] E. Voce, The Relationship between Stress and Strain for homogeneous deformation, Journal of the Institute of Metals, 74 (1948) 537-562.

[66] C. Tomé, G.R. Canova, U.F. Kocks, N. Christodoulou, J.J. Jonas, The relation between macroscopic and microscopic strain hardening in F.C.C. polycrystals, Acta Metallurgica, 32 (1984) 1637-1653.

[67] M. Knezevic, R.J. McCabe, C.N. Tomé, R.A. Lebensohn, S.R. Chen, C.M. Cady, G.T. Gray Iii, B. Mihaila, Modeling mechanical response and texture evolution of $\alpha$-uranium as a function of strain rate and temperature using polycrystal plasticity, International Journal of Plasticity, 43 (2013) 70-84. 
[68] M. Ardeljan, R.J. McCabe, I.J. Beyerlein, M. Knezevic, Explicit incorporation of deformation twins into crystal plasticity finite element models, Computer Methods in Applied Mechanics and Engineering, 295 (2015) 396-413.

[69] M. Knezevic, R.J. McCabe, R.A. Lebensohn, C.N. Tomé, B. Mihaila, Finite element implementation of a self-consistent polycrystal plasticity model: Application to $\alpha$-uranium, Proceedings: Volume 2: Materials Properties, Characterization, and Modeling TMS (The Minerals, Metals \& Materials Society), in, 2012, pp. 789-796.

[70] M. Knezevic, R.J. McCabe, R.A. Lebensohn, C.N. Tomé, C. Liu, M.L. Lovato, B. Mihaila, Integration of self-consistent polycrystal plasticity with dislocation density based hardening laws within an implicit finite element framework: Application to low-symmetry metals, Journal of the Mechanics and Physics of Solids, 61 (2013) 2034-2046.

[71] S. Mahesh, C.N. Tomé, R.J. McCabe, G.C. Kaschner, A. Misra, I.J. Beyerlein, Application of a substructure-based hardening model to copper under loading path changes, Metallurgical and Materials Transactions A, 35 (2004) 3763-3774.

[72] M. Knezevic, B. Drach, M. Ardeljan, I.J. Beyerlein, Three dimensional predictions of grain scale plasticity and grain boundaries using crystal plasticity finite element models, Computer Methods in Applied Mechanics and Engineering, 277 (2014) 239-259.

[73] M. Jahedi, M. Ardeljan, I.J. Beyerlein, M.H. Paydar, M. Knezevic, Enhancement of orientation gradients during simple shear deformation by application of simple compression, Journal of Applied Physics, 117 (2015) 214309.

[74] M. Jahedi, M. Knezevic, M. Paydar, High-Pressure Double Torsion as a Severe Plastic Deformation Process: Experimental Procedure and Finite Element Modeling, J. of Materi Eng and Perform, 24 (2015) 1471-1482.

[75] M. Jahedi, M.H. Paydar, M. Knezevic, Enhanced microstructural homogeneity in metalmatrix composites developed under high-pressure-double-torsion, Materials Characterization, 104 (2015) 92-100.

[76] R.G. Anderson, J.W. Bishop, The effect of neutron irradiation and thermal cycling on permanent deformations in uranium under load, in: Symposium on uranium and graphite, The Institute of Metals, London 1962, pp. 17-23.

[77] E.S. Fisher, H.J. McSkimin, Adiabatic elastic moduli of single crystal alpha Uranium, Journal of Applied Physics, 29 (1958) 1473 - 1484.

[78] I.J. Beyerlein, R.J. McCabe, C.N. Tome, Stochastic processes of $\{1012\}$ deformation twinning in hexagonal close-packed polycrystalline zirconium and magnesium, International Journal for Multiscale Computational Engineering, 9 (2011) 459-480.

[79] I.J. Beyerlein, R.J. McCabe, C.N. Tomé, Effect of microstructure on the nucleation of deformation twins in polycrystalline high-purity magnesium: A multi-scale modeling study, Journal of the Mechanics and Physics of Solids, 59 (2011) 988-1003.

[80] H. Mecking, U.F. Kocks, Kinetics of flow and strain-hardening., Acta Metallurgica et Materialia, 29 (1981) 1865-1875.

[81] U.F. Kocks, H. Mecking, Physics and phenomenology of strain hardening: the FCC case, Progress in Materials Science, 48 (2003) 171-273.

[82] M. Jahedi, M. Ardeljan, I.J. Beyerlein, M.H. Paydar, M. Knezevic, Enhancement of orientation gradients during simple shear deformation by application of simple compression, Journal of applied physics, (2015). 
[83] M. Zecevic, M. Knezevic, A dislocation density based elasto-plastic self-consistent model for the prediction of cyclic deformation: Application to Al6022-T4, International Journal of Plasticity, 72 (2015) 200-217.

[84] M. Knezevic, J.S. Carpenter, M.L. Lovato, R.J. McCabe, Deformation behavior of the cobalt-based superalloy Haynes 25: Experimental characterization and crystal plasticity modeling, Acta Materialia, 63 (2014) 162-168.

[85] M. Knezevic, I.J. Beyerlein, M.L. Lovato, C.N. Tomé, A.W. Richards, R.J. McCabe, A strain-rate and temperature dependent constitutive model for BCC metals incorporating nonSchmid effects: Application to tantalum-tungsten alloys, International Journal of Plasticity, 62 (2014) 93-104.

[86] A. Bhattacharyya, M. Knezevic, M. Abouaf, Characterization of Crystallographic Texture and Intra-Grain Morphology in Cross-Rolled Tantalum, Metallurgical and Materials Transactions A, 46 (2015) 1085-1096.

[87] M. Ardeljan, I.J. Beyerlein, M. Knezevic, A dislocation density based crystal plasticity finite element model: Application to a two-phase polycrystalline HCP/BCC composites, Journal of the Mechanics and Physics of Solids, 66 (2014) 16-31.

[88] M. Knezevic, T. Nizolek, M. Ardeljan, I.J. Beyerlein, N.A. Mara, T.M. Pollock, Texture evolution in two-phase $\mathrm{Zr} / \mathrm{Nb}$ lamellar composites during accumulative roll bonding, International Journal of Plasticity, 57 (2014) 16-28.

[89] M. Ardeljan, M. Knezevic, T. Nizolek, I.J. Beyerlein, N.A. Mara, T.M. Pollock, A study of microstructure-driven strain localizations in two-phase polycrystalline HCP/BCC composites using a multi-scale model, International Journal of Plasticity, 74 (2015) 35-57.

[90] J.S. Carpenter, T. Nizolek, R.J. McCabe, M. Knezevic, S.J. Zheng, B.P. Eftink, J.E. Scott, S.C. Vogel, T.M. Pollock, N.A. Mara, I.J. Beyerlein, Bulk texture evolution of nanolamellar Zr$\mathrm{Nb}$ composites processed via accumulative roll bonding, Acta Materialia, 92 (2015) 97-108.

[91] I.J. Beyerlein, C.N. Tomé, A dislocation-based constitutive law for pure $\mathrm{Zr}$ including temperature effects, International Journal of Plasticity, 24 (2008) 867-895.

[92] R. Madec, B. Devincre, L. Kubin, T. Hoc, D. Rodney, The Role of Collinear Interaction in Dislocation-Induced Hardening, Science, 301 (2003) 1879-1882.

[93] M.A. Meyers, K.K. Chawla, Mechanical Behavior of Materials., Prentice Hall, Upper Saddle River, New Jersey, 1998.

[94] L. Capolungo, I.J. Beyerlein, C.N. Tomé, Slip-assisted twin growth in hexagonal closepacked metals, Scripta Materialia, 60 (2009) 32-35.

[95] G. Proust, C.N. Tomé, G.C. Kaschner, Modeling texture, twinning and hardening evolution during deformation of hexagonal materials, Acta Materialia, 55 (2007) 2137-2148.

[96] E.S. Fisher, Temperature dependence of the elastic moduli in alpha uranium single crystals, part IV (298 to $923 \mathrm{~K}$ ), Journal of Nuclear Materials, 18 (1966) 39-54.

[97] R.I. Borja, Plasticity Modeling \& Computation, Springer Berlin Heidelberg, 2013. 